\title{
High resolution cryo-EM structure of the EspA filament from EPEC: revealing the mechanism of effector translocation in the type 3 secretion system
}

\author{
B Lyons ${ }^{1}$ \\ ${ }^{1}$ University of British Columbia, Vancouver, BC \\ lyonsb819@gmail.com
}

Contributors: Bronwyn Lyons, Claire Atkinson, Wanyin Deng, Antonio Serapio Palacios, Brett Finlay, Natalie C.J. Strynadka Antimicrobial resistance (AMR) is a growing concern for the global population, predominantly in animal husbandry, hospitals, and developing countries. Several pathogens are of particular concern, including Pseudomonas aeruginosa and Enterobacter spp. (Escherichia coli). The rise in AMR has contributed to the decline in development of novel antibiotics, thus pushing the world closer to a pre-antibiotic era. Virulence mechanisms that bacteria use are often not required for their survival and therefore are promising targets for the development of anti-virulence compounds. The type III secretion system (T3SS) is a highly conserved virulence mechanism employed by several Gram-negative pathogens, such as those discussed above. The T3SS is a syringe-like proteinaceous channel that spans the inner and outer membranes of the bacterial cell, projecting into the extracellular medium where it interacts with the host cell membrane to deliver proteinaceous virulence factors. The main components of the T3SS are: (1) the basal body, spanning the inner and outer membranes of the bacterial cell; (2) the needle that projects from the basal body and secretin lumen, and (3) the tip and pore-forming translocon that completes the continuous channel from bacteria to host. Entero-pathogenic and -hemorrhagic E. coli (EPEC/EHEC) possess a distinct T3SS from that of other species; EPEC/EHEC possess a needle extension, or filament, in place of the 'tip' component. This filament, called EspA, can be upwards of $700 \mathrm{~nm}$ in length, extending the channel from the needle through EspA and connecting to the translocon upon host-cell contact. It is thought that the EspA filament may provide a more flexible extension of the conduit to protect effectors in the unfavourable environment of the gut epithelium. The function of EspA is absolutely required for colonization of EPEC within the gut epithelium and espA EPEC knockouts are associated with decreased virulence. Here we describe the cryo-EM structure of natively-sheared EspA filaments from EPEC, determined to 3.6 A resolution. Data were collected at the High-Resolution Macromolecular Electron Microscopy (HRMEM) facility at the University of British Columbia. The EspA protomer is described as a coiled-coil with each helix connected by an insertion domain that decorates the exterior of the filament. Within the filament lumen, a pattern of positively charged residues adjacent to a hydrophobic groove lines the lumen of the filament in a spiral manner, suggesting a mechanism of translocation through the channel mediated via electrostatics. Using structure-guided mutagenesis of these residues, in vivo studies corroborate the role of these residues in secretion function. Interestingly, the EspA filament is distinct from the needle structure, in that it possesses a helix-turn-strand insertion that connects the two helices of the conserved coiled-coil rather than a short loop as seen in the recent Salmonella PrgI needle structure, or a globular domain as seen in other non-filamentous orthologs. The high-resolution structure of the EspA filament will aid in structureguided drug design of novel antivirulence therapeutics. This may lead to improved prophylactic treatment of EHEC/EPEC infections, which are on the rise due to increased produce contamination.

Acta Cryst. (2020). A76, a97 